\title{
Energy dependence of the efficiency of high-energy negatively charged particle beam deflection by planar channeling in a bent crystal
}

\author{
I.V. Kyryllin ${ }^{1,2, a}$, N.F. Shul'ga ${ }^{1,2}$ \\ ${ }^{1}$ Akhiezer Institute for Theoretical Physics, National Science Center "Kharkov Institute of Physics and Technology", Akademicheskaya Str., 1, \\ Kharkiv 61108, Ukraine \\ ${ }^{2}$ V.N. Karazin Kharkiv National University, Svobody Sq. 4, Kharkiv 61022, Ukraine
}

Received: 23 September 2019 / Accepted: 28 November 2019 / Published online: 17 December 2019

(C) The Author(s) 2019

\begin{abstract}
The study of planar channeling of high-energy negatively charged particles in bent crystals was carried out. The value of the critical radius of planar channeling in the Doyle-Turner approximation for the atomic potential is determined. The dependence of the maximum angle at which a given fraction of beam particles could be deflected by means of planar channeling in a bent crystal on the particle energy was found. We identified the ideal parameters for the exploitation of planar channeling for negatively charged particle beam deflection by planar channeling in a bent crystal at current and future high-energy accelerators, e.g., SLAC, MAMI, ILC or muon colliders.
\end{abstract}

\section{Introduction}

When a high-energy charged particle moves in a crystal at a small angle to one of the main crystalline axes or planes, its trajectory is determined mainly by the field of continuous potential of the crystalline atomic strings or planes [1]. In a bent crystal, such motion in the field of bent atomis strings or bent atomic planes makes it possible to deflect charged particles from their initial direction of motion. The possibility of using a bent crystal to deflect high-energy charged particles was first proposed in Refs. [2] and [3]. In these works, the deflection of particles that move in a bent crystal in the planar channeling mode was proposed [1]. It was assumed that if charged particles move in a potential well formed by the adjacent atomic planes of a bent crystal, these particles will deflect, following the bending of crystalline atomic planes. An illustration of planar channeling in a bent crystal is shown in Fig. 1. In this figure solid line shows the potential energy of an electron in the field of bent atomic planes. The interplanar

a e-mail: kirillin@kipt.kharkov.ua distance is indicated as $d_{\mathrm{p}}$. If the orthogonal energy of the particle $E_{\perp}$ [1] is less than the height of the potential barrier between the planes, the particle channels in a bent crystal (this case is illustrated by a dotted line). Otherwise, if the orthogonal energy of the particle exceeds the height of the potential barrier between the planes, the particle is above-barrier and is not captured in the planar channeling mode (this case is illustrated by a dash-dotted line). Experimentally, this deflection mechanism for positively charged particles was confirmed in Ref. [4]. For positively charged particles, the potential well formed by the neighboring atomic planes of the crystal, lies between the planes, while for the negatively charged particles the position of the center of potential well coincides with the position of the atomic plane. For this reason dechanneling caused by incoherent scattering of charged particles (which occurs primarily because of the thermal vibrations of the atoms of the crystals and scattering by the electron subsystem of atoms) is more intense for negatively charged particles than for positively charged ones. This is why planar channeling in a bent crystal is more effective for positively charged particles.

Experimental confirmation of the possibility of using planar channeling in a bent crystal to deflect negatively charged particles was obtained in Ref. [5]. The deflection efficiency in that experiment was approximately $30 \%$. In recent years, a number of experiments have been carried out to study the planar channeling of negatively charged particles in bent crystals [6-10]. These experiments demonstrated the deflection of a part of the electron beam in different energy ranges of particles from $855 \mathrm{MeV}$ to $150 \mathrm{GeV}$.

In this paper, we carry out a theoretical analysis of planar channeling of negatively charged particles in a bent crystal, discuss the optimal crystal parameters for observing the deflection of negatively charged particles and analyze the 


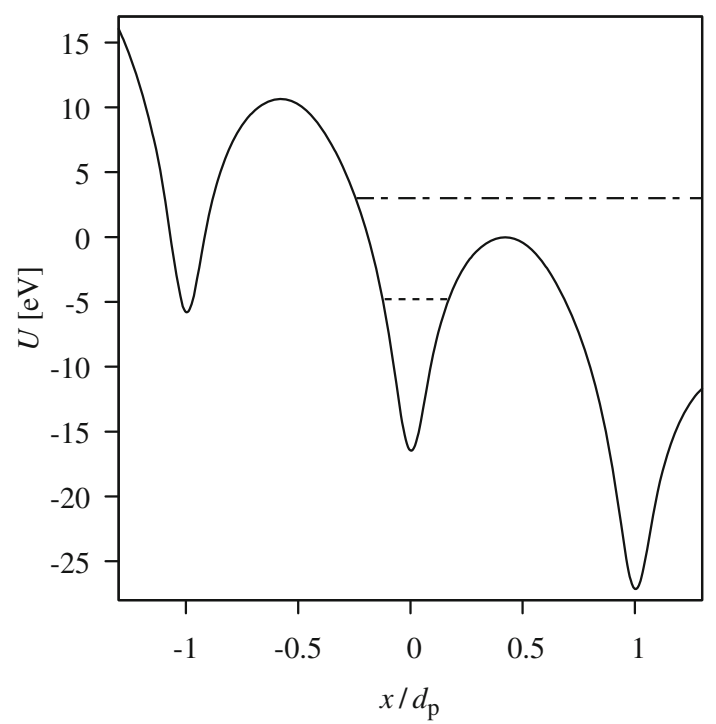

Fig. 1 An illustration of interplanar potential in a bent crystal. Dotted line corresponds to planar channeling, while dash-dotted line corresponds to above-barrier motion

energy dependence of the efficiency of high-energy negatively charged particle beam deflection by planar channeling in a bent crystal. This analysis is important because bent crystals can be applied for extraction or collimation of high-energy negatively charged particle beams on existing and future accelerators, e.g., SLAC, MAMI, ILC or muon colliders.

\section{Calculations in parabolic approximation of planar potential}

In the article [11], on the basis of an analytical calculation and a numerical simulation of high-energy negatively charged particle motion in a bent crystal, planar channeling in a bent crystal was studied. Namely, the dependence of the deflection efficiency on the radius of crystal curvature was analyzed. It was shown that if one wants to deflect a given part of the beam particles there exists an optimal radius of crystal curvature at which this given fraction will be deflected on the maximum angle. This radius of curvature was called the optimal radius of crystal curvature $R_{\text {opt }}$. For analytical calculations in the article [11] the parabolic approximation of planar potential was used:

$$
\begin{aligned}
U_{\mathrm{p}}(x)= & -\frac{U_{0}}{d_{\mathrm{p}}^{2}}\left[\left(2 x+d_{\mathrm{p}}\right)^{2} H\left(-x\left(d_{\mathrm{p}}+x\right)\right)\right. \\
& \left.+\left(2 x-d_{\mathrm{p}}\right)^{2} H\left(x\left(d_{\mathrm{p}}-x\right)\right)\right],
\end{aligned}
$$

$|x| \leq d_{\mathrm{p}} / 2$, where $d_{\mathrm{p}}$ is the distance between neighboring atomic planes, $U_{0}$ is the potential well depth and $H(\zeta)$ is the Heaviside step function [12] which equals to 1 for $\zeta>0$, to
0 for $\zeta<0$ and to $\frac{1}{2}$ for $\zeta=0$. In [10] it was shown that such a simple parabolic approximation gives almost the same result for estimating the fraction of negatively charged particles, that becomes under-barrier when the beam impinges on a crystal, as a more complex and realistic Doyle-Turner approximation [13].

This simple model of planar potential gave a possibility to obtain an analytical expression for the maximum angle up to which a given fraction of beam particles $f$ remains underbarrier and thus could be deflected by means of planar channeling in a bent crystal with radius of curvature $R$ :

$\alpha_{f}=\frac{\theta_{\mathrm{c}}^{2}}{2 \xi^{2} R\left(\operatorname{erf}^{-1}\left(\frac{f}{1-\sqrt{R_{\mathrm{c}} / R}}\right)\right)^{2}}$,

where $\theta_{\mathrm{c}}$ is the critical angle for planar channeling [1], $R_{\mathrm{c}}$ is the critical radius of planar channeling and $\operatorname{erf}^{-1}(\zeta)$ is the inverse error function, $\xi$ is defined as follows: if $\vartheta$ is the angle between the crystal planes and momentum of the particle, and $\vartheta_{0}$ is the standard deviation of the distribution of $\vartheta$ angles of beam particles at the given thickness of the crystal $l$, then $\vartheta_{0}=\xi \sqrt{l}$.

If we want to study the dependence of $\alpha_{f}$ and $R_{\mathrm{opt}}$ from the kinetic energy of particles $E$ we should notice that $R_{\mathrm{c}}=\frac{E d_{\mathrm{p}}}{4 U_{0}}$, $\theta_{\mathrm{c}}=\sqrt{\frac{2 U_{0}}{E}}\left(1-\frac{R_{c}}{R}\right)[14]$ and by analogy with multiple scattering in amorphous medium we will assume that $\xi \propto$ $1 / E$. Now if we introduce a variable $\rho=R / R_{\mathrm{c}}$, we will be able to write (2) as

$\alpha_{f}=\frac{U_{0}}{E \xi^{2} R_{\mathrm{c}}} \frac{\left(1-\rho^{-1}\right)^{2}}{\rho\left(\operatorname{erf}^{-1}\left(\frac{f}{1-\rho^{-1 / 2}}\right)\right)^{2}}$.

As $R_{\mathrm{c}} \propto E$ and $\xi \propto 1 / E$, the first factor in (3) does not depend on $E$.

The optimal radius of curvature which corresponds to the $\max \left(\alpha_{f}\right)$ can be found by solving the equation

$\left.\frac{d \alpha_{f}}{d R}\right|_{\rho=\rho_{\mathrm{opt}}}=0$

This means that the value of $\rho_{\text {opt }}$ in parabolic approximation of planar potential does not depend on $E$ and so $R_{\text {opt }} \propto E$.

\section{Simulation in Doyle-Turner approximation of planar potential}

The model of parabolic potential of atomic planes is useful for analytical calculations, however this model does not take into account a number of properties of real inter-planar potential. Now we will find the dependence of $\alpha_{f}$ and $R_{\text {opt }}$ on the kinetic energy of particles in more realistic Doyle-Turner approximation of planar potential. In this approximation the 
potential energy of a particle with a charge, that equals to the charge of an electron, in the field of an atomic string could be written as

$U_{\mathrm{str}}(\rho)=-\frac{8 \pi^{2} \hbar^{2}}{m_{\mathrm{e}} d} \sum_{k=1}^{4} \frac{\alpha_{k}}{\beta_{k}+B} e^{-\frac{4 \pi^{2} \rho^{2}}{\beta_{k}+B}}$,

where $m_{\mathrm{e}}$ is an electron mass, $d$ is the distance between neighboring atoms in the atomic string, $\alpha_{k}$ and $\beta_{k}$ are coefficients found in [13] for a large number of elements, $B=8 \pi^{2}\left\langle r_{T}{ }^{2}\right\rangle$ and $r_{T}$ is the rms atomic thermal vibration amplitude in one direction $\left(r_{T} \approx 0.075 \AA\right.$ for $\mathrm{Si}$ at $\left.293 \mathrm{~K}\right), \rho=\sqrt{x^{2}+y^{2}}$ is the distance from the atomic string. After averaging of (5) over $y$ one can obtain the potential energy of a particle with a charge, that equals to the charge of an electron, in the field of an atomic plane as

$U_{\mathrm{pl}}(x)=-\frac{4 \pi^{\frac{3}{2}} \hbar^{2}}{m_{\mathrm{e}} d d_{\mathrm{s}}} \sum_{k=1}^{4} \frac{\alpha_{k}}{\sqrt{\beta_{k}+B}} e^{-\frac{4 \pi^{2} x^{2}}{\beta_{k}+B}}$,

where $d_{\mathrm{s}}$ is the distance between neighboring atomic strings in the atomic plane.

To obtain the potential of atomic planes in a crystal in the Doyle-Turner approximation one must sum up potentials of atomic planes (6). Since $U_{\mathrm{pl}}(x)$ in Eq. (6) decreases rapidly with increasing distance from the atomic plane, only a limited number of neighboring atomic planes determine the value of the potential at the selected point inside the crystal. This fact gives us a possibility to sum the potentials of atomic planes analytically and find the potential energy of a high-energy charged particle with a charge that equals to the charge of an electron as

$U_{\mathrm{p}}(x)=\sum_{n=-\infty}^{\infty} U_{\mathrm{pl}}\left(x-x_{n}\right)$,

where points $x_{n}$ correspond to the location of atomic planes. After summation one can obtain

$U_{\mathrm{p}}(x)=-\frac{2 \pi \hbar^{2}}{m_{\mathrm{e}} d d_{\mathrm{s}} d_{\mathrm{p}}} \sum_{k=1}^{4} \alpha_{k} \theta_{3}\left(\pi \frac{x}{d_{\mathrm{p}}}, e^{-\frac{\beta_{k}+B}{4 d_{\mathrm{p}}^{2}}}\right)$,

where $\theta_{3}(u, q)=\sum_{n=-\infty}^{\infty} q^{n^{2}} e^{2 n u i}$ is the Jacobi theta function of the third kind [15], $i^{2}=-1$.

Equation (8) was found in assumption that atomic planes are equidistant. This is correct for (100) and (110) atomic planes of Si crystal. For (111) atomic planes, if we consider one of them, the distance to right neighboring plane is not the same as distance to left neighboring plane. If we denote the distance between odd atomic planes (that is equal to the distance between even atomic planes) as $d_{\mathrm{p} 2}$ and the distance between two nearest planes as $d_{\mathrm{p} 1}$, potential energy of a highenergy charged particle with a charge that equals to the charge
Table 1 Maximum values of the plane potential derivative

\begin{tabular}{llll}
\hline Plane & $U_{\mathrm{p}}^{\prime}\left(x_{0}\right)(\mathrm{eV} / \AA)$ & $U_{0}(e V)$ & $4 U_{0} / d_{\mathrm{p}}(\mathrm{eV} / \AA)$ \\
\hline$(100)$ & 39.5225 & 11.7894 & 34.7340 \\
$(110)$ & 57.3313 & 21.3573 & 44.4934 \\
$(111)$ & 66.8021 & 28.3173 & 48.1676 \\
\hline
\end{tabular}

of an electron in the field of (111) planes of Si crystal can be written as

$$
\begin{aligned}
U_{\mathrm{p}}(x)= & -\frac{2 \pi \hbar^{2}}{m_{\mathrm{e}} d d_{\mathrm{s}} d_{\mathrm{p} 2}} \sum_{k=1}^{4} \alpha_{k}\left[\theta_{3}\left(\pi \frac{x}{d_{\mathrm{p} 2}}, e^{-\frac{\beta_{k}+B}{4 d_{\mathrm{p} 2}^{2}}}\right)\right. \\
& \left.+\theta_{3}\left(\pi \frac{x-d_{\mathrm{p} 1}}{d_{\mathrm{p} 2}}, e^{-\frac{\beta_{k}+B}{4 d_{\mathrm{p} 2}^{2}}}\right)\right] .
\end{aligned}
$$

In a bent crystal, however, particle moves in the so-called effective potential [16]:

$U_{\text {eff }}(x)=U_{\mathrm{p}}(x)+E x / R$.

Let us find the critical radius of curvature of the crystal in the Doyle-Turner approximation of planar potential. The critical radius of curvature of the crystal means the radius at which potential wells in effective potential (10) disappear. This means that at $R=R_{\mathrm{c}}$ the derivative of $U_{\mathrm{eff}}(x)$ is nonnegative. So, to find $R_{\mathrm{c}}$ one must find the point $x_{0}$ at which the second derivative of $U_{\text {eff }}(x)$ is equal to zero and solve an equation

$\left.\frac{d U_{\mathrm{eff}}(x)}{d x}\right|_{x=x_{0}}=0$.

And thus we obtain

$R_{\mathrm{c}}=\frac{E}{\left|U_{\mathrm{p}}^{\prime}\left(x_{0}\right)\right|}$.

In Table 1 we calculated in the Doyle-Turner approximation of planar potential values of $U_{\mathrm{p}}^{\prime}\left(x_{0}\right)$ and the potential well depth $U_{0}$ in a straight crystal for three main planar orientations of a Si crystal. For (111) orientation calculations were done for a deeper potential well.

Now we can compare $R_{\mathrm{c}}$ obtained in parabolic $\left(R_{\mathrm{c}}=\frac{E d_{\mathrm{p}}}{4 U_{0}}\right)$ and Doyle-Turner approximation of planar potential. To do this in Table 1 we calculated values of $\frac{4 U_{0}}{d_{\mathrm{p}}}$ for three main planar orientations of a Si crystal. Comparison between values of $U_{\mathrm{p}}^{\prime}\left(x_{0}\right)$ and $4 U_{0} / d_{\mathrm{p}}$ show that for all three main planar orientations of a Si crystal parabolic approximation gives overestimated values for the critical radius of curvature of the crystal. The difference in values of $R_{\mathrm{c}}$ increases linearly with increasing energy. In Fig. 2 we show the difference in values of the critical radius of curvature calculated in parabolic and Doyle-Turner approximation of planar potential on example of (110) atomic planes of a Si crystal. One can see that 


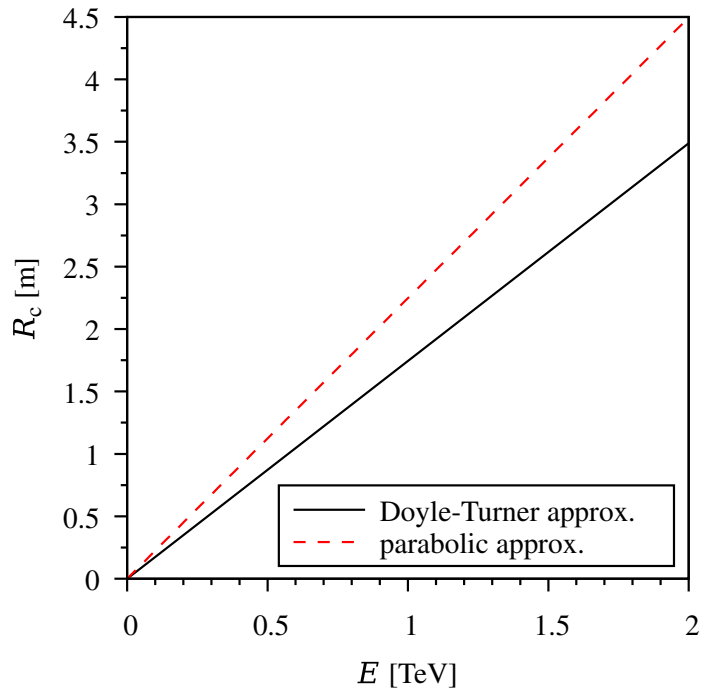

Fig. 2 The dependence of the critical radius of curvature of the crystal on particle energy in parabolic and Doyle-Turner approximation of planar potential

although at low energies the difference is small, it grows linearly with increasing particle energy and at an energy of $2 \mathrm{TeV}$, the difference reaches $1 \mathrm{~m}$.

Unfortunately, the expression (8) is too complex to obtain the dependence of $\alpha_{f}$ and $R_{\text {opt }}$ on particle energy analytically, as we did in the previous section for parabolic potential. However, this dependence could be found with a help of numerical simulation. We carried out a numerical simulation of $\pi^{-}$-mesons motion in a bent $\mathrm{Si}$ crystal. The simulation was carried out for particles with energy from $10 \mathrm{GeV}$ to $10 \mathrm{TeV}$. The simulation code was the same as in Refs. $[17,18]$. The code computes the trajectory of a high-energy charged particle in the field of continuous atomic string potential in the Doyle-Turner approximation through numerical integration of the equation of motion. It takes into account the incoherent scattering caused by thermal vibrations of atoms and scattering on electrons. Other kinds of incoherent scattering were not taken into account considering the small crystal thickness. In the simulation the crystal was oriented in such a way that impinging particles were moving in bent (110) planar channels.

For each considered particle energy value we simulated propagation of the beam of $10^{5}$ particles through the crystal of a given radius of curvature $R$. The initial angular divergence of the beam was equal to zero. For each particle we calculated the thickness of the crystal at which particle dechanneled. This gave us a possibility to find maximum deflection angle $\alpha_{f}$ on which the given fraction $f$ of beam particles remains underbarrier and thus can be deflected by a bent crystal with the given $R$. Then we were changing the radius of curvature of the crystal and repeated simulation. Thanks to this procedure, we were able to find the dependence of $\alpha_{f}$ on $R$. The example

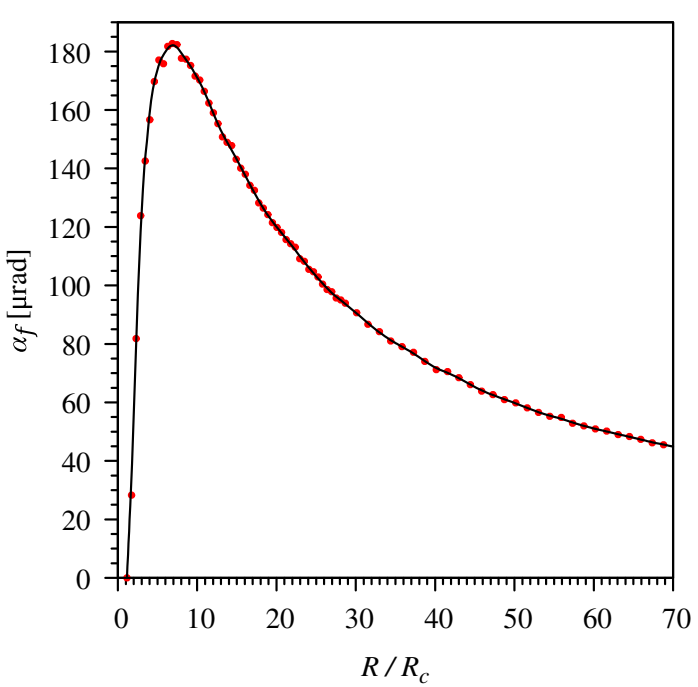

Fig. 3 The dependence of the maximum deflection angle $\alpha_{f}$ on which the given fraction $f=0.1$ of beam particles can be deflected by a bent crystal on the radius of curvature of the crystal

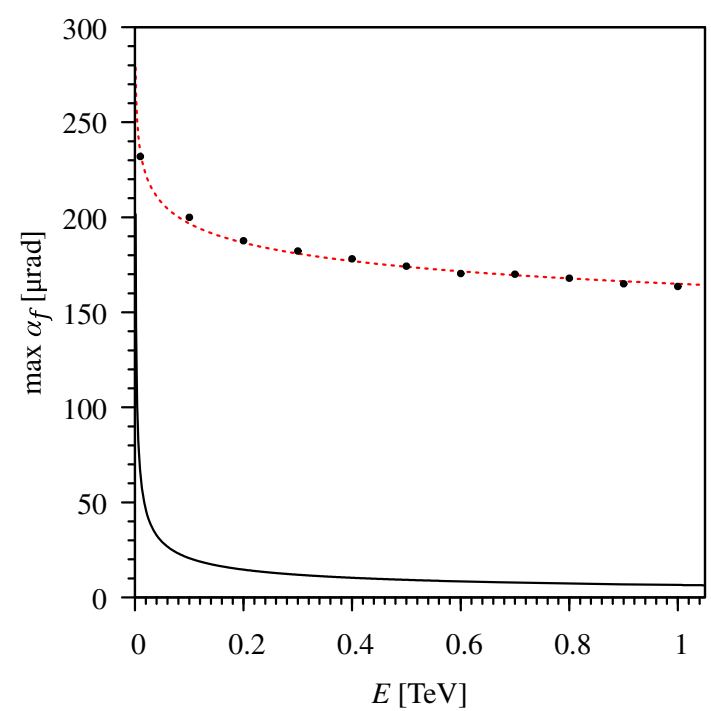

Fig. 4 The dependence of the maximum angle by which the fraction of particles $f=0.1$ can be deflected on the particle energy. The black solid curve shows the dependence of the critical angle of the planar channeling on the particle energy

of such dependence is shown in Fig. 3 for $E=300 \mathrm{GeV}$ and $f=0.1$. In this figure one can see a well-defined maximum at $R / R_{\mathrm{c}} \in(6,7)$ which coincides with the results obtained in [11].

After repeating the procedure described in the previous paragraph, we obtained the values of the $\max \alpha_{f}$ and $R_{\text {opt }}$ for a number of particle energies in the range from $10 \mathrm{GeV}$ to $10 \mathrm{TeV}$. The values of $\max \alpha_{f}$ obtained by simulation are shown in Fig. 4 by black dots for $f=0.1$. Red dashed curve shows a fit of simulated points by function $g(x)=A x^{m}$. For the obtained simulation results $A \approx 165 \mu \mathrm{rad}$ and 


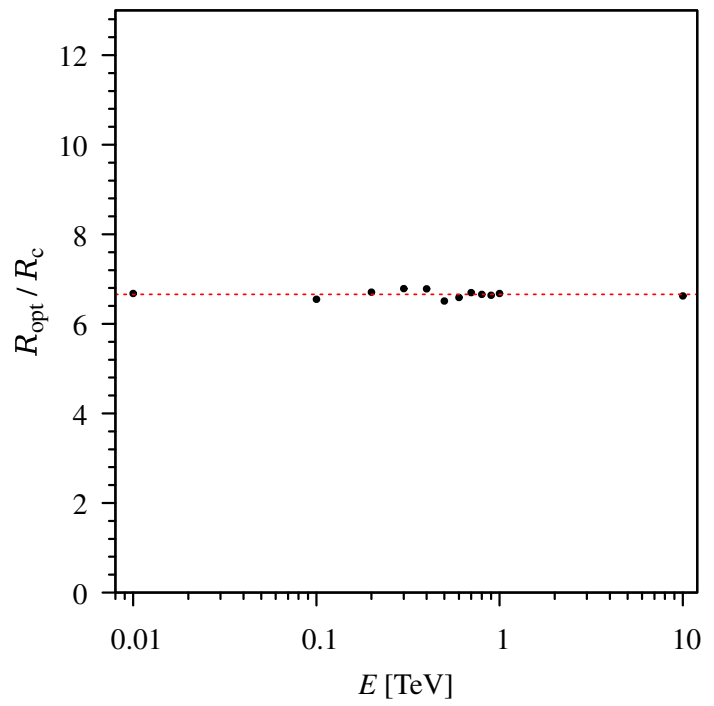

Fig. 5 The dependence of the ratio of the optimal radius of curvature to the critical radius of planar channeling on the particle energy for $f=0.1$

$m \approx-0.076$. It can be seen that the approximating curve very accurately describes the distribution of points obtained by simulation. The black solid curve in the figure shows the dependence of the critical angle of the planar channeling on the particle energy. It can be seen that the maximum deflection angle, by which $10 \%$ of the particles of a beam of negatively charged particles can be deflected using planar channeling, is many times higher than the critical angle of planar channeling.

In Fig. 5 we depicted the dependence of the ratio of the optimal radius of curvature to the critical radius of planar channeling on the particle energy for $f=0.1$. Simulation results are shown by black points, while the mean value $\left\langle R_{\text {opt }} / R_{\mathrm{c}}\right\rangle \approx 6.7$ is shown by red dashed line. Simulation results are in a coincidence with theoretical results obtained in [11]. It can be seen that the ratio of the optimal radius of curvature to the critical radius of planar channeling is independent of the particle energy. Taking into account formula (12), we conclude that the optimal radius of planar channeling increases linearly with increasing particle energy.

In Fig. 6 we shown the dependence of $\max \alpha_{f}$ on the particle energy for $f=0.1$ in double logarithmic scale. As in Fig. 4 black dots show simulation results, while the red dashed line shows a fit of simulated points by function $g(x)=A x^{m}$ (the values of the fitting parameters are indicated above). The black solid line in the figure shows the dependence of the critical angle of the planar channeling on the particle energy. The fact that the points obtained by simulation on a double logarithmic scale fit on a straight line shows that $\max \alpha_{f}$ depends on the particle energy according to a power law.

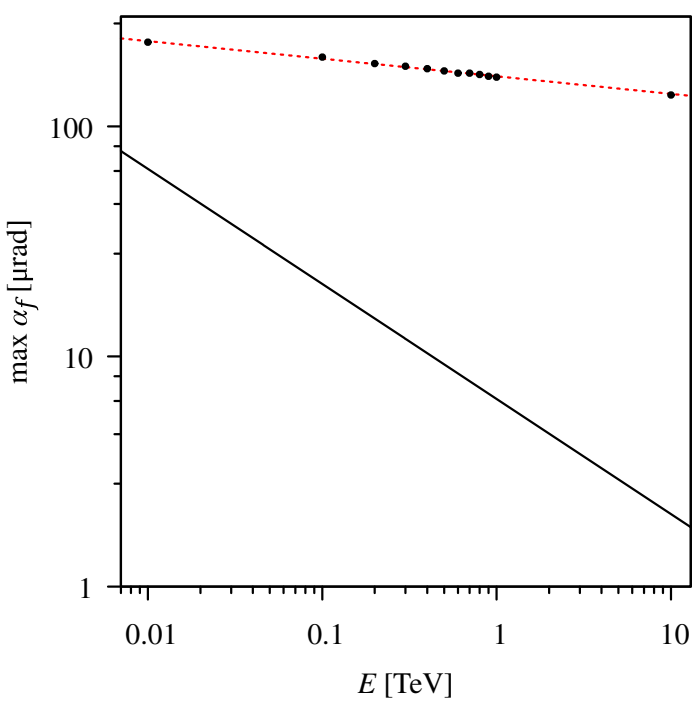

Fig. 6 The dependence of the maximum angle by which the fraction of particles $f=0.1$ can be deflected on the particle energy on a double logarithmic scale. The black solid line shows the dependence of the critical angle of the planar channeling on the particle energy

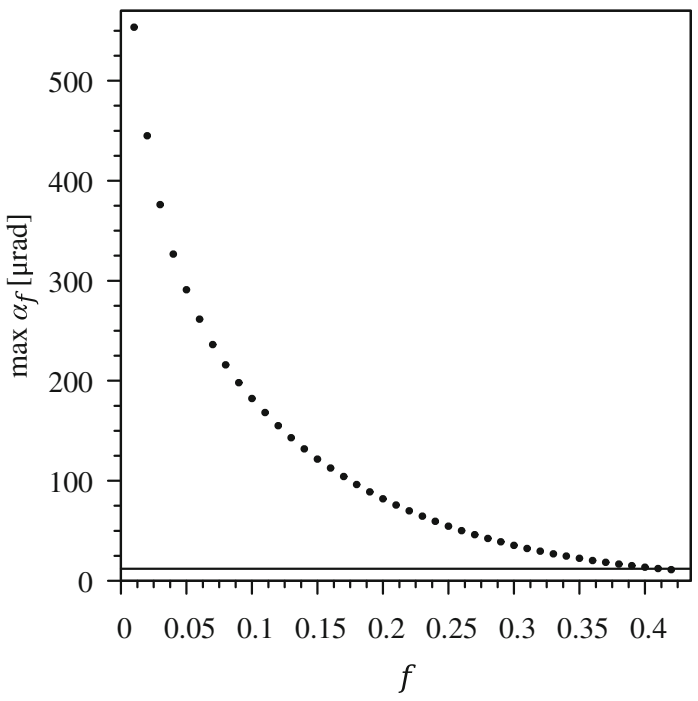

Fig. 7 The dependence of the maximum angle by which the given fraction of particles can be deflected on $f$ for $E=300 \mathrm{GeV}$. The line shows the value of the critical angle of planar channeling

It is important to note that, in accordance with formula (3), the value of $\alpha_{f}$ substantially depends on the fraction of beam particles that must be deflected by a bent crystal. In Fig. 7 we shown this dependence for $E=300 \mathrm{GeV}$. Simulation results are depicted by dots, while line shows the value of the critical angle of planar channeling. One can see that with a decrease in $f$ the $\max \alpha_{f}$ is growing rapidly. However, it should be noted that planar channeling makes it possible to deflect only a smaller part of the beam of negatively charged particles. And the smaller this part, the more it can be deflected. 


\section{Conclusions}

In summary, an investigation on the mechanism of planar channeling of high-energy negatively charged particles in bent crystals was carried out. The consideration showed that there is a maximum in the dependence of the deflection from the radius of curvature of the crystal. This maximum corresponds to the optimal radius of planar channeling. It was shown that this optimal radius grows linearly with increasing energy. It was also shown that maximum deflection angle $\max \alpha_{f}$ on which the given fraction $f$ of beam particles can be deflected by a bent crystal decreases with increasing energy according to a power law. Moreover, the analysis of the dependence of $\max \alpha_{f}$ on parameter $f$ shown that $\max \alpha_{f}$ grows rapidly with a decrease in $f$. The study showed that planar channeling in a bent crystal makes it possible to deflect part of the beam of negatively charged by angles that significantly exceed the critical angle of planar channeling. It was shown that only a smaller part of the beam particles can be deflected at a large angle.

Acknowledgements We recognized the support of the National Academy of Sciences of Ukraine (budget program "Support for the Development of Priority Areas of Scientific Research" (6541230), project C-2/50-2019).

Data Availability Statement This manuscript has no associated data or the data will not be deposited. [Authors' comment: This is a theoretical study and no experimental data has been listed. All simulation data generated during this study are included in this published article.]

Open Access This article is licensed under a Creative Commons Attribution 4.0 International License, which permits use, sharing, adaptation, distribution and reproduction in any medium or format, as long as you give appropriate credit to the original author(s) and the source, provide a link to the Creative Commons licence, and indicate if changes were made. The images or other third party material in this article are included in the article's Creative Commons licence, unless indicated otherwise in a credit line to the material. If material is not included in the article's Creative Commons licence and your intended use is not permitted by statutory regulation or exceeds the permitted use, you will need to obtain permission directly from the copyright holder.To view a copy of this licence, visit http://creativecomm ons.org/licenses/by/4.0/.

Funded by SCOAP ${ }^{3}$.

\section{References}

1. J. Lindhard, Danske Vid. Selsk. Mat. Fys. Medd. 34, 14 (1965)

2. E.N. Tsyganov, Preprint Fermilab TM-682 (1976)

3. E.N. Tsyganov. Preprint Fermilab TM-684 (1976)

4. A.F. Elishev, N.A. Filatova et al., Phys. Lett. B 88, 387 (1979)

5. W. Scandale et al., Phys. Lett. B 681, 233 (2009)

6. W. Scandale et al., Phys. Lett. B 719, 70 (2013)

7. A. Mazzolari et al., Phys. Rev. Lett. 112, 135503 (2014)

8. U. Wienands et al., Phys. Rev. Lett. 114, 074801 (2015)

9. L. Bandiera et al., Phys. Rev. Lett. 115, 025504 (2015)

10. T.N. Wistisen et al., Phys. Rev. Accel. Beams 19, 071001 (2016)

11. I.V. Kirillin, Phys. Rev. Accel. Beams 20, 104401 (2017)

12. M. Abramowitz, I.A. Stegun, Handbook of Mathematical Functions with Formulas, Graphs, and Mathematical Tables (United States Department of Commerce, National Bureau of Standards, New York, 1972)

13. P.A. Doyle, P.S. Turner, Acta Crystallogr. A 24, 390 (1968)

14. V.M. Biryukov, Y.A. Chesnokov, V.I. Kotov, Crystal Channeling and Its Application at High-Energy Eccelerators (Springer, Berlin, 1997)

15. I.S. Gradshteyn, I.M. Ryzhik, Table of Integrals, Series, and Products, 7th edn. (Academic Press, London, 2007)

16. A.I. Akhiezer, N.F. Shul'ga et al., Physics-Uspekhi 38, 1119 (1995)

17. N.F. Shul'ga, I.V. Kirillin, V.I. Truten', Phys. Lett. B 702, 100 (2011)

18. N.F. Shul'ga, I.V. Kirillin, V.I. Truten', Nuovo Cim. C 34, 425 (2011) 